\title{
Stochastic rotational stability of tower cranes under gusty winds
}

\author{
H. Vanvinckenroye \\ Structural Engineering Division, Faculty of Applied Sciences, University of Liège, Liège, Belgium \\ V. Denoël \\ Structural Engineering Division, Faculty of Applied Sciences, University of Liège, Liège, Belgium
}

\begin{abstract}
This work aims to study the rotational stability of a tower crane left free to rotate. Indeed, in case of important wind velocities, small oscillations can increase and build up into autorotations due to autoparametric excitation of the structure. Many references in the literature describe the limit between oscillation and autorotation for simple cases like the deterministic pendulum and evidence the importance of the Hamiltonian of a system on its stability. In this context the susceptibility of the structure to this dynamical instability is characterized by the average time necessary to reach a given energy barrier departing from an initial energy level. This first passage time is the solution of the Pontryagin equation and is approached by an asymptotic expansion. First- and second-order terms are calculated as well as the boundary layer solution providing a correction when the initial energy is close to the barrier level.
\end{abstract}

\section{INTRODUCTION}

Tower cranes are designed to transport heavy weights. Over the last years, a large number of crane collapses has been recorded and massively reported in the media, e.g. in New-York in 2012 under storm Sandy (Figure 1). Many others are available on dedicated websites (Christie, 2012) and (Mok, 2008).

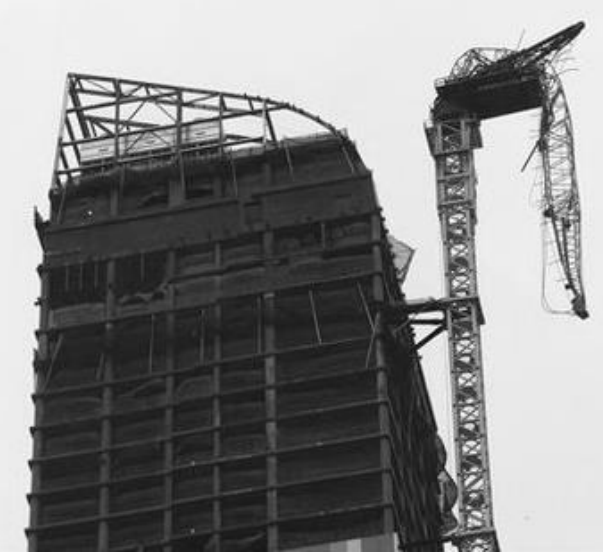

Figure 1. Crane failure in New-York, 2012 (Christie, 2012).

Although the behavior of cranes is a widely studied problem in the literature, most research works focus on the study of structures in use, i.e. during lift operations. The dynamic behavior of a crane lifting weights is studied by Ghigliazza by analogy with a pendulum with moving support (Ghigliazza \&
Holmes, 2002) while the planning and modeling of the path traveled by the crane and its load are studied in (Sakawa \& Nakazumi, 1985) and (Hamalainen, et al., 1995) respectively. As tower cranes are high-rise and lattice structures, wind is an important excitation. In case of high wind velocities, the crane is out-of-service and left free to rotate as a weathervane in order to avoid overturning of the structure. The corresponding out-of-service wind speed is studied in (Eden, et al., 1981), (Eden, et al., 1983) and (Sun, et al., 2009). In opposition with all the previous research about tower cranes in use, Voisin performed experimental analysis in order to understand and characterize other crane instabilities under wind excitation and determine the susceptibility of a tower crane to autorotation when it is out-ofservice. This experiment consists in the determination of a probability of autorotation of the jib in a given environment. This method allows to validate or not a configuration by experimental campaigns (Voisin, 2003) and (Voisin, et al., 2004).

A wide variety of tools already exists concerning wind loading, stochastic processes, dynamic analysis of structures and can be combined to analyze and better understand the behavior of tower cranes in a stochastic wind velocity field. A conceptual model of this problem will help catching the impact of the different geometrical, structural and wind parameters and their effect on autorotation. In this perspective, the crane is represented by a single degree-offreedom model composed of a rigid jib rotating around a fixed pivot (Vanvinckenroye, 2015). This 
mechanism is similar to a pendulum. As the wind loading depends on the angular position and velocity of the crane through aerodynamic forces and pressure coefficients, the wind excitation is called autoparametric which is a characteristic of the pendulum as well. Assuming no damping, the dimensionless governing equation of this system submitted to an external force $w(t)$ and a parametric force $u(t)$ is given by:

$\ddot{x}+(1+u(t)) \sin x=w(t)$

Poulin studies the evolution of unstable regions for an excitation that varies continuously from periodic to stochastic (Poulin \& Flierl, 2008). A wide variety of excitations are specifically tested in the literature. Gitterman studies the stability and the period of the pendulum under deterministic and stochastic excitation of its support in (Gitterman, 2010a) and (Gitterman, 2010b). He observes that an increasing stochasticity of the excitation induces larger, but of lower energy unstable ranges of parameters.

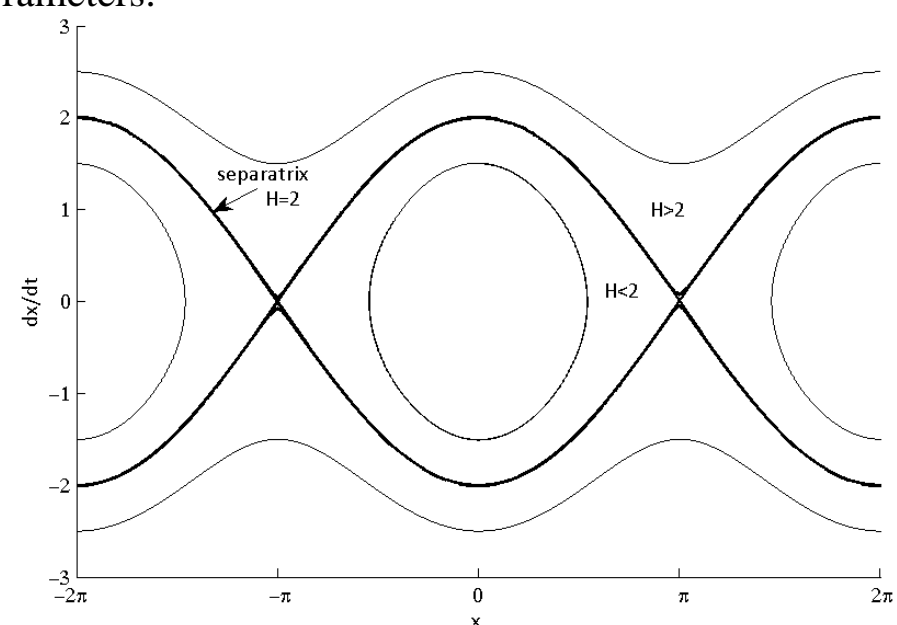

Figure 2. Phase portrait of the non-excited Mathieu equation (Gitterman, 2010b).

The simplest case of a pendulum submitted to the vertical gravitational acceleration provides the energy curves presented in Figure 2. Assuming no damping, the pendulum will describe oscillations or rotations depending on its energy level $H$. Bishop, Garira, $\mathrm{Xu}$ and Clifford developed analytical solutions to approach the separatrix thanks to the harmonic balance method, the perturbation method and the critical velocity criterion (Garira \& Bishop, 2003), (Clifford \& Bishop, 1994), (Bishop \& Clifford, 1996) and (Xu \& Wiercigroch, 2006). Narrow band and random phase excitations are investigated by Alevras and Yurchenko in (Yurchenko, et al., 2013) and (Alevras, et al., 2013) through a numerical path integration providing stability lobes when the support is submitted to a vertical harmonic excitation. Xu presents similar results in $(\mathrm{Xu}$, et al., 2005) for a harmonically excited pendulum by evidencing the basins of attraction in the phase plane.
(Mallick \& Marcq, 2004) present an analytical method providing an expression for the asymptotic probability distribution function of the energy.

This work aims to study the susceptibility of a crane to reach this instability zone when excited by Gaussian white noise excitations $u(t)$ and $w(t)$. If the stability is governed by the energy, i.e. the system is quasi-Hamiltonian, the probability of instability can be studied as the time needed to reach that critical energy level. As the excitation is stochastic, the first passage time is a random variable. An asymptotic expansion method was developed by Moshchuk in (Moshchuk, et al., 1995a) and (Moshchuk, et al., 1995b) to approximate the mean first passage time of a nonlinear stochastic process representing a ship motion on random sea waves. Chunbiao and Liu developed in respectively (Chunbiao \& Bohou, 2000) and (Liu, et al., 2013) the stochastic averaging method for quasi-nonintegrable-hamiltonian systems submitted to Gaussian and Poisson white noises and $\mathrm{Li}$ extended this approach to stochastic fractional derivative systems with power-form restoring force in ( $\mathrm{Li}$, et al., 2015). The first passage time can also be estimated through a multi-level Monte Carlo algorithm (Primozic, 2011). In this work, the asymptotic expansion is developed for the linearized form of relation (1) taking the form of a stochastic Mathieu equation:

$\ddot{x}+(1+u(t)) x=w(t)$

The quasi-Hamiltonian system is characterized by a set of Itô differential equations (Schuss, 2010) and the first passage time is obtained by solution of the corresponding Pontryagin equation (Moshchuk, et al., 1995a) and (Moshchuk, et al., 1995b). Finally, the accuracy of the expansion is illustrated by comparison with Monte Carlo simulations of the motion.

\section{AVERAGE FIRST PASSAGE TIME}

The problem is governed by equation (2) where $u(t)$ and $w(t)$ are Brownian white noises of low intensity $S_{u}$ and $S_{W}$, and $x$ is the rotational position of the crane. This problem can be represented in the state space by its Itô formulation:

$d \boldsymbol{x}=\boldsymbol{f}(\boldsymbol{x}, t) d t+\boldsymbol{b}(\boldsymbol{x}, t) d \boldsymbol{B}$

where $\boldsymbol{x}=\left(\begin{array}{c}q \\ p\end{array}\right), \boldsymbol{f}=\left(\begin{array}{c}p \\ -q\end{array}\right), \boldsymbol{b}=\left(\begin{array}{cc}0 & 0 \\ -q & 1\end{array}\right)$ and $\boldsymbol{B}=\left(\begin{array}{l}B_{u} \\ B_{w}\end{array}\right)$ is the vector of Brownian motions $u$ and $W$ characterized by the intensity matrix

$\boldsymbol{S}=\left(\begin{array}{cc}S_{u} & 0 \\ 0 & S_{w}\end{array}\right)=\varepsilon \boldsymbol{v}=\varepsilon\left(\begin{array}{cc}v_{u} & 0 \\ 0 & \nu_{w}\end{array}\right)$

As the white noise excitation is small, the system is quasi-Hamiltonian, which means that the Hamiltonian of the system given by 
$\mathrm{H}=\frac{\mathrm{p}^{2}}{2}+\frac{q^{2}}{2}$

varies slowly in time.

The unperturbed system describes a closed trajectory of constant energy $H$ called a homoclinic orbit. This motion presents a period

$T=2 \int_{q_{1}}^{q_{2}} \frac{d q}{\dot{q}}=2 \int_{-\sqrt{2 H}}^{\sqrt{2 H}} \frac{1}{\sqrt{2 H-q^{2}}} d q=2 \pi$.

The average first passage time through a level of energy $H_{c}$ from an initial energy level $H$ is the exit time $U(\boldsymbol{x})$ from a region $D=\left\{(x, \dot{x}) \mid H(x, \dot{x}) \leq H_{c}\right\}$ and satisfies the Pontryagin partial differential equation (7):

$L U(\boldsymbol{x})=-1$

where $L$ is the backward Kolmogorov operator and is given by (Schuss, 2010):

$L[\cdot]=\frac{1}{2} \boldsymbol{T} \boldsymbol{r}\left\{\left[\frac{\partial}{\partial x} \frac{\partial}{\partial x}(\cdot)\right] \boldsymbol{\sigma}\right\}+\boldsymbol{f}(\boldsymbol{x}, t) \frac{\partial \cdot}{\partial x}$

where $\boldsymbol{\sigma}=\boldsymbol{\varepsilon} \boldsymbol{b}(\boldsymbol{x}, t) \boldsymbol{v} \boldsymbol{b}^{T}(\boldsymbol{x}, t)$. This operator can be decomposed in two operators:

$L[\cdot]=L_{1}[\cdot]+\epsilon L_{2}[\cdot]$.

with

$\left\{\begin{array}{l}L_{1}[\cdot]=p \frac{\partial \cdot}{\partial q}-q \frac{\partial \cdot}{\partial p} \\ L_{2}[\cdot]=\frac{1}{2}\left(q^{2} v_{u}+v_{w}\right) \frac{\partial^{2}}{\partial p^{2}}\end{array}\right.$

The first operator represents the derivative along the direction of the conservative system, i.e. along the homoclinic $H$.

The asymptotic expansion method developed by Moshchuk in (Moshchuk, et al., 1995a) and (Moshchuk, et al., 1995b) solves equation (7) for an approximate form of the first passage time:

$U(p, q) \sim U_{n}(p, q)+G_{n}(p, q)$

where $U_{n}$ is the regular asymptotic outer solution and is of the form

$U_{n}(p, q)=\frac{1}{\epsilon} u_{0}(p, q)+u_{1}(p, q)+\cdots+\epsilon^{n-1} u_{n}(p, q)$

and $G_{n}$ stands for the inner solution in the boundary layer at the limit of the domain $D$ so that $L U_{n}=-1$ and $L G_{n}=0$. This boundary layer solution will be developed with the second-order term $u_{1}$.

Collecting terms of likewise powers of $\varepsilon$ in relation (7) yields:

$L_{1} u_{0}=0$

$L_{1} u_{1}+L_{2} u_{0}=-1$

$L_{1} u_{2}+L_{2} u_{1}=0$

\subsection{Leading order solution}

The leading order equation (13a) means that $u_{0}$ is constant along each homoclinic orbit and is consequently a function of the Hamiltonian $H$ only.
The averaging along a period of Equation (13b) provides the information to determine $u_{O}(H)$. Indeed, as the homoclinic orbits are closed, averaging along this curve gives zero and equation (13b) becomes

$\left\langle L_{2} u_{0}\right\rangle=-1$

$\frac{1}{2}\left[\left\langle q^{2} v_{u}+v_{w}\right\rangle \frac{d u_{0}}{d H}+\left\langle p^{2}\left(q^{2} v_{u}+v_{w}\right)\right\rangle \frac{d^{2} u_{0}}{d H^{2}}\right]=-1$

where the following relations have been used for the partial derivatives:

$u_{0}=u_{0}(H) \rightarrow \begin{cases}\frac{\partial u_{0}}{\partial p}= & p \frac{d u_{0}}{d H} \\ \frac{\partial^{2} u_{0}}{\partial p^{2}}= & \frac{d u_{0}}{d H}+p^{2} \frac{d^{2} u_{0}}{d H^{2}}\end{cases}$

and the operator $\langle\cdot\rangle$ represents the average over one period of the unperturbed motion:

$\langle\cdot\rangle=\frac{1}{2 \pi} \int_{0}^{2 \pi} \cdot d t$.

The averaged second-order Pontryagin equation therefore reads:

$\left(\frac{H}{2} v_{u}+\frac{1}{2} v_{w}\right) \frac{d u_{0}}{d H}+\left(\frac{H^{2}}{4} v_{u}+\frac{H}{2} v_{w}\right) \frac{d^{2} u_{0}}{d H^{2}}=-1$,

with the boundary conditions $u_{0}\left(H_{c}\right)=0$ and $|U(0)|<\infty$, equation (17) provides the following solution:

$\mathrm{u}_{0}(H)=\frac{4 \epsilon}{S_{u}} \log \left(\frac{H_{c} S_{u}+2 S_{w}}{H S_{u}+2 S_{w}}\right)$.

This solution presents two limit formulations when $u(t)$ and $w(t)$ are respectively equal to zero,

$S_{u} \rightarrow 0: u_{0} \rightarrow 2 \epsilon \frac{H_{c}-H}{S_{w}}$

$S_{w} \rightarrow 0: u_{0} \rightarrow 4 \epsilon \frac{\log \left(H_{c}\right)-\log (H)}{S_{u}}$

\subsection{Second order solution}

As the leading order solution $u_{0}$ is known, equation (13b) is used to determine the second order component $u_{1}$

The variables $q$ and $p$ are changed into energyphase variables $k$ and $\theta$ assuming following expressions:

$\left\{\begin{array}{l}p=2 k \cos \theta \\ q=2 k \sin \theta\end{array}\right.$

so that the Hamiltonian $H$ is now equal to $2 k^{2}$.

Itô formulation (3) taking into account the WongZakai correction terms $\delta_{i}$ detailed in (Schuss, 2010), becomes:

$d \boldsymbol{x}^{*}=\boldsymbol{f}^{*}(\boldsymbol{x}, t) d t+\boldsymbol{b}^{*}(\boldsymbol{x}, t) d \boldsymbol{B}$

where $d \boldsymbol{x}^{*}=\left(\begin{array}{c}d k \\ d \theta\end{array}\right), \boldsymbol{f}^{*}=\left(\begin{array}{c}\sigma_{22} \frac{\partial^{2} k}{\partial p^{2}} \\ 1+\sigma_{22} \frac{\partial^{2} \theta}{\partial p^{2}}\end{array}\right)=\left(\begin{array}{c}\delta_{1} \\ 1+\delta_{2}\end{array}\right)$, 
$\boldsymbol{b}^{*}=\left(\begin{array}{cc}-k \cos \theta \sin \theta & \frac{\cos \theta}{2} \\ \sin ^{2} \theta & -\frac{\sin \theta}{2 k}\end{array}\right)$ and the operators

$L_{1}$ and $L_{2}$ become thanks to $\boldsymbol{\sigma}^{*}=\boldsymbol{\varepsilon} \boldsymbol{b}^{*}\left(\boldsymbol{x}^{*}, t\right) \boldsymbol{v} \boldsymbol{b}^{* T}\left(\boldsymbol{x}^{*}, t\right)$ :

$\left\{\begin{array}{l}L_{1}[\cdot]=\frac{\partial \cdot}{\partial \theta} \\ L_{2}[\cdot]=\delta_{1} \frac{\partial \cdot}{\partial k}+\delta_{2} \frac{\partial \cdot}{\partial \theta}+\frac{1}{2} \sigma_{11}^{*} \frac{\partial^{2} \cdot}{\partial k^{2}}+\frac{1}{2} \sigma_{22}^{*} \frac{\partial^{2} \cdot}{\partial \theta^{2}}+\sigma_{12}^{*} \frac{\partial^{2} .}{\partial k \partial \theta}\end{array}\right.$ sion:

Equation (13b) provides the following expres-

$L_{1} u_{1}=-1-L_{2} u_{0}=\left\langle L_{2} u 0\right\rangle-L_{2} u 0$

$=k^{2} v_{u} \cos 2 \theta \frac{d u_{0}}{d H}+\left(k^{2} \cos 4 \theta v_{u}-\cos 2 \theta v_{w}\right) \frac{d^{2} u_{0}}{d H^{2}}$

Integration of expression (23) with respect to $\theta$ provides a decomposition of $u_{1}$ into two components with the constant of integration $u_{12}(\mathrm{k})$ :

$u_{1}(k, \theta)=u_{11}(k, \theta)+u_{12}(k)$

with

$u_{11}(k, \theta)=\frac{k^{2} S_{u} \sin 2 \theta}{2\left(k^{2} S_{u}+S_{w}\right)^{2}}\left((\cos 2 \theta-2) k^{2} S_{u}-3 S_{w}\right)$.

The averaging of equation (13c) over one period of the unperturbed motion provides:

$\left\langle L_{1} u_{2}\right\rangle+\left\langle L_{2} u_{12}\right\rangle+\left\langle L_{2} u_{12}\right\rangle=0$

$\left\langle L_{2} u_{12}\right\rangle=\frac{1}{16}\left(k^{2} v_{u}+v_{w}\right) \frac{d^{2} u_{12}}{d k^{2}}+\frac{3 k^{2} v_{u}+v_{w}}{16 k} \frac{d u_{12}}{d k}=0$,

and $\mathrm{u}_{12}(\mathrm{k})$ takes the following form:

$u_{12}(k)=\frac{C_{1} \epsilon}{S_{w}} \log \left(\frac{k^{2} \epsilon}{k^{2} S_{u}+S_{w}}\right)+C_{2}$

The first constant of integration $C_{1}$ is equal to zero in order to respect the solvability condition $|U(0)|<\infty$. The second constant $C_{2}$ will be determined together with the boundary layer solution in order to respect the boundary conditions.

\subsection{Boundary layer}

The boundary layer equation is:

$L G_{n}=0$

The coordinate stretching is classical in the boundary layers problems (Denoël \& Detournay, 2010). The boundary layer solution $G_{n}$ is written as a function of the stretched coordinate $\xi=\left(H-H_{c}\right) / \sqrt{ } \mathcal{E}$,

$G_{n}(\xi, \theta)=g_{1}(\xi, \theta)+\sqrt{\epsilon} g_{2}(\xi, \theta)+\cdots+\epsilon^{\frac{n-1}{2}} g_{n}(\xi, \theta)$.

Similarly, the operator $L$ is transformed via de Taylor expansion of the functions $\sigma_{i}^{*}$ and $\delta_{i}$ in the neighborhood of $H=H_{c}$ :

$\delta_{i}(H, \theta)=\delta_{i}\left(H_{c}, \theta\right)+\sqrt{\epsilon} \xi \delta_{i}^{(1)}\left(H_{c}, \theta\right)+\cdots$

$\sigma_{i}^{*}(H, \theta)=\sigma_{i}^{*}\left(H_{c}, \theta\right)+\sqrt{\epsilon} \xi \sigma_{i}^{*(1)}\left(H_{c}, \theta\right)+\cdots$

The backward Kolmogorov operator becomes:

$L[\cdot]=\frac{\partial \cdot}{\partial \theta}+4 H_{c} \sigma_{1}^{*}\left(H_{c}, \theta\right) \frac{\partial^{2}}{\partial \xi^{2}}$

$$
\begin{gathered}
+\sqrt{\epsilon}\left[\left[2 \sqrt{2 H_{c}} \delta_{1}\left(H_{c}, \theta\right)+2 \sigma_{1}^{*}\left(H_{c}, \theta\right)\right] \frac{\partial \cdot}{\partial \xi}\right. \\
+2 \sqrt{2 H_{c}} \sigma_{12}^{*}\left(H_{c}, \theta\right) \frac{\partial^{2} \cdot}{\partial \xi \partial \theta} \\
\left.+4 H_{c} \xi \sigma_{1}^{*(1)}\left(H_{c}, \theta\right) \frac{\left.\partial^{2} \cdot\right]}{\partial \xi^{2}}\right]+\cdots \\
=\Lambda_{0}[\cdot]+\sqrt{\epsilon} \Lambda_{1}[\cdot]+\epsilon \Lambda_{2}[\cdot]+\cdots
\end{gathered}
$$

so that the governing equation (28) becomes:

$$
\begin{aligned}
L G_{n} & =\left(\Lambda_{0}+\sqrt{\epsilon} \Lambda_{1}+\cdots\right)\left[g_{1}+\sqrt{\epsilon} g_{2}+\cdots\right] \\
& =\Lambda_{0} g_{1}+\sqrt{\epsilon}\left[\Lambda_{0} g_{2}+\Lambda_{1} g_{1}\right]+\cdots=0
\end{aligned}
$$

Balancing again the similar powers of $\varepsilon$ provides the expression of the functions $g_{i}(\xi, \theta)$. In particular, the first order solution $g_{1}(\xi, \theta)$ is the solution of the following diffusion equation:

$\Lambda_{0} g_{1}=\frac{\partial g_{1}}{\partial \theta}+4 H_{c} \sigma_{1}^{*}\left(H_{c}, \theta\right) \frac{\partial^{2} g_{1}}{\partial \xi^{2}}=0$

with the boundary conditions $g_{1}(0, \theta)=-u_{1}\left(H_{c}, \theta\right)$ and $g_{1}(\xi, \theta) \rightarrow 0$ when $\xi \rightarrow-\infty$. The solution of this equation is given by (Moshchuk, et al., 1995b):

$$
\begin{aligned}
g_{1}(\xi, \theta)=\mathrm{b}_{0} & +\sum_{n=1}^{\propto} b_{n} e^{\sqrt{\frac{n c_{1}}{2}} \xi} \cos \left(n \alpha(\theta)-\sqrt{\frac{n c_{1}}{2}} \xi\right) \\
& +\sum_{n=1}^{\infty} a_{n} e^{\sqrt{\frac{n c_{1}}{2} \xi}} \sin \left(n \alpha(\theta)-\sqrt{\frac{n c_{1}}{2}} \xi\right)
\end{aligned}
$$

with

$$
\begin{aligned}
c_{1} & =2 \pi / \int_{0}^{T} 4 H_{c} \sigma_{1}^{*}\left(H_{c}, s\right) d s \\
\alpha(\theta) & =c_{1} \int_{0}^{\theta} 4 H_{c} \sigma_{1}^{*}\left(H_{c}, s\right) d s \\
b_{0} & =-\frac{1}{2 \pi} \int_{0}^{2 \pi} u_{1}\left(H_{c}, \theta(\alpha)\right) d \alpha \\
b_{n} & =-\frac{1}{\pi} \int_{0}^{2 \pi} u_{1}\left(H_{c}, \theta(\alpha)\right) \cos (n \alpha) d \alpha \\
a_{n} & =-\frac{1}{\pi} \int_{0}^{2 \pi} u_{1}\left(H_{c}, \theta(\alpha)\right) \sin (n \alpha) d \alpha
\end{aligned}
$$

Because of the second boundary condition, it follows that $b_{0}=0$ :

$-\frac{1}{2 \pi} \int_{0}^{2 \pi} u_{1}\left(H_{c}, \theta(\alpha)\right) d \alpha=0$.

Finally, averaging (24) with respect to variable $\alpha$, the constant of integration $C_{2}$ is obtained:

$$
\left\langle u_{12}(k)\right\rangle=C_{2}=-\frac{1}{2 \pi} \int_{0}^{2 \pi} u_{11}\left(H_{c}, \alpha\right) d \alpha=0
$$

Adapted to relation (35), only the even coefficients $a_{n}$ are non zero.

All in all, the solution including the first two terms is obtained as

$U=\frac{1}{\epsilon} u_{0}+u_{11}+u_{12}+g_{1}$

with $u_{0}, u_{11}$ and $u_{12}$ given by (18), (25) et (27). 


\section{RESULTS AND CONCLUSION}

Figure 3 presents the average first passage time of the energy barrier $H_{c}=0.1$ as a function of the initial energy level for three different white noise intensities of $u$ and $w$. If $S_{u}=0$, the relation is linear while it is logarithmic if $S_{W}=0$. It can be observed from relations (17) and (24) that the effects of $u$ and $W$ on the first passage time have the same order of magnitude if $S_{u}$ has the same order as $H_{c} S_{W}$. This is confirmed in Figure 3. The results obtained with 2,000 Monte Carlo simulations are very close to the analytical result obtained with first- and second-order expansion terms.

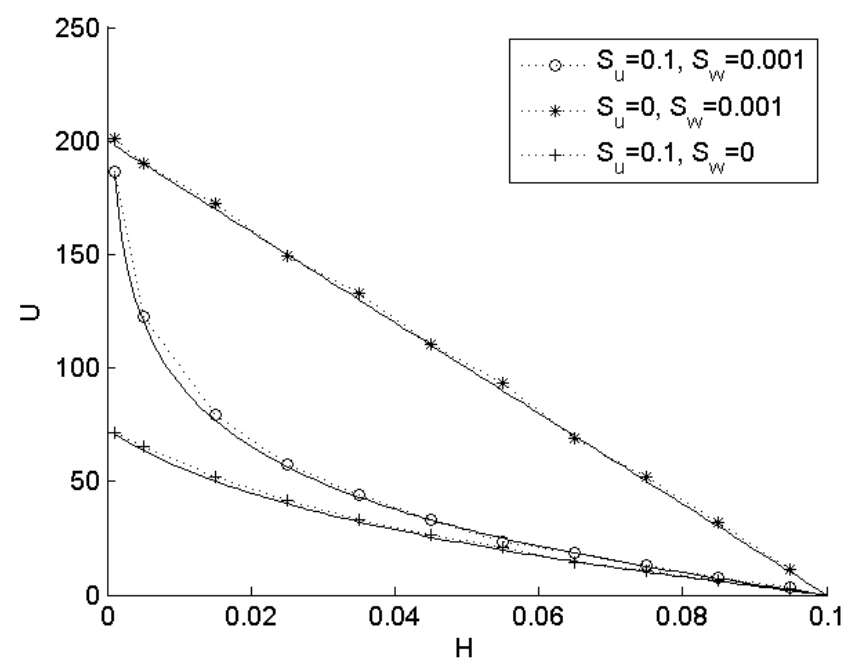

Figure 3. Comparison of theoretical (solid line) and simulated (dotted line) first-passage times with $H_{c}=0.1$ and $\theta=3 \pi / 4$.

Figure 4 represents the contributions of the second-order term $u_{1}$ as well as the boundary layer $g_{1}$. The second order term has a contribution independent on the energy level $\mathrm{H}$ and the boundary layer is a correction on the second order term close to $H=H_{c}$ that keeps a first passage time equal to zero at this point.

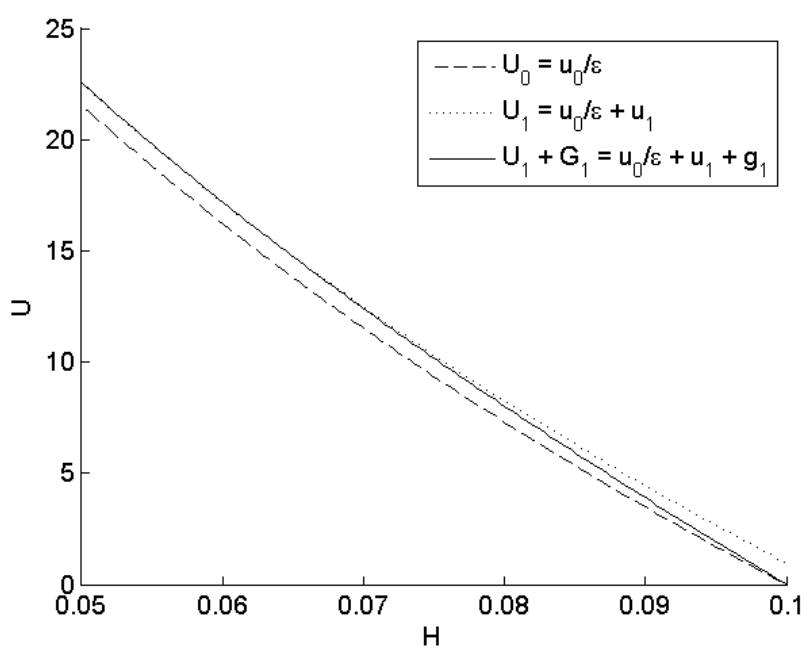

Figure 4. Comparison of the first-order, second-order and boundary layer terms with with $S_{u}=0, S_{w}=0.001, H_{c}=0.1$ and $\theta=3 \pi / 4$.
Finally, Figure 5 presents the boundary layer contribution in the $(H, \theta)$-plane. The correction vanishes when the initial energy level $H$ is getting away from the barrier $H_{c}$. Close to the barrier, the correction is either positive or negative depending on the initial phase $\theta$.

As a conclusion, the second order expansion provides a concluding approximation of the first passage time. Second order terms are of small order but the boundary layer contribution ensures the respect of the limit conditions.

(Vanvinckenroye, 2015)

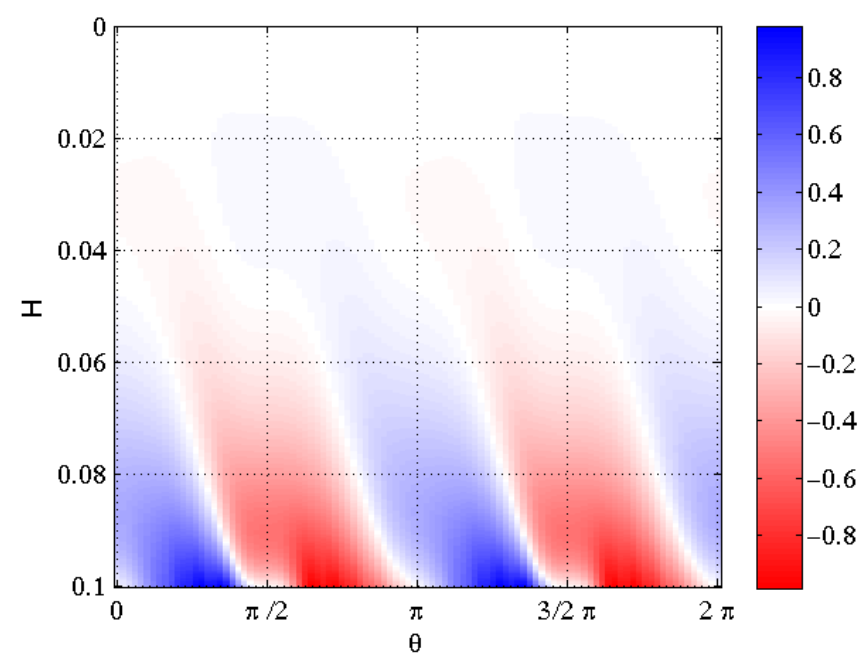

Figure 5. Evolution of the boundary layer solution $g_{1}$, with $S_{u}=0, S_{w}=0.001$ and $H_{c}=0.1$.

\section{REFERENCES}

Alevras, P., Yurchenko, D. \& Naess, A., 2013. Numerical investigation of the parametric pendulum under filtered random phase excitation.

Bishop, S. \& Clifford, M., 1996. Zones of chaotic behaviour in the parametrically excited pendulum. Journal of Sound and Vibration, 189(1), pp. 142147.

Christie, L., 2012. craneaccidents.com. http://www.craneaccidents.com/2012/11/report/upda te/one 57s-crane-problem/.

Chunbiao, G. \& Bohou, X., 2000. First-passagetime of quasi-non-integrable-hamiltonian system. Acta Mechanica Sinica, 16(2), pp. 183-192.

Clifford, M. \& Bishop, S., 1994. Approximating the Escape Zone for the Parametrically Excited Pendulum. Journal of Sound and Vibration, 172(4), pp. 572-576.

Denoël, V. \& Detournay, E., 2010. Multiple scales solution for a beam with a small bending stiffness. Journal of Engineering Mechanics, 136(1), pp. 69-77. 
Eden, J., Butler, A. \& Patient, J., 1983. Wind tunnel tests on model crane structures. Engineering Structures, Volume 5, pp. 289-298.

Eden, J., Iny, A. \& Butler, A., 1981. Cranes in storm winds. Engineering Structures, Volume 3, pp. 175-180.

Garira, W. \& Bishop, S., 2003. Rotating solutions of the parametrically excited pendulum. Journal of Sound and Vibration, 263(1), pp. 233-239.

Ghigliazza, R. \& Holmes, P., 2002. On the dynamics of cranes, or spherical. International Journal of Non-Linear Mechanics, 37(7), pp. 12111221.

Gitterman, M., 2010a. Spring pendulum: Parametric excitation vs an external force. Physica A: Statistical Mechanics and its Applications, 389(16), pp. 3101-3108.

Gitterman, M., 2010b. The Chaotic Pendulum. New Jersey: World Scientific Publishing.

Hamalainen, J., Virkkunen, J., Baharova, L. \& Marttinen, A., 1995. Optimal path planning for a trolley crane: fast and smooth transfer of load. IEEE Proceedings - Control Theory and Applications, Volume 142, pp. 51-57.

Liu, W., Zhu, W. \& Xu, W., 2013. Stochastic stability of quasi non-integrable Hamiltonian systems under parametric excitations of Gaussian and Poisson white noises. Probabilistic Engineering Mechanics, Volume 32, pp. 39-47.

Li, W. et al., 2015. First passage of stochastic fractinoal derivative systems with power-form restoring force.. International Journal of Non-Linear Mechanics, Volume 71, pp. 83-88.

Mallick, K. \& Marcq, P., 2004. On the stochastic pendulum with Ornstein-Uhlenbeck noise. Journal of Physics A: Mathematical and General, 37(17), p. 14.

Mok, K., 2008. Youtube.com. YouTube - Crane Spinning out of Control. https://www.youtube.com/watch? v=0olg_j3289Q.

Moshchuk, N., Ibrahim, R., Khasminskii, R. \& Chow, P., 1995a. Asymptotic expansion of ship capsizing in random sea waves - I. First-order approximation. Int. J. Non-Linear Mechanics, 30(5), pp. 727-740.

Moshchuk, N., Khasminskii, R., Ibrahim, R. \& Chow, P., 1995b. Asymptotic expansion of ship capsizing in random sea waves - II. Second-order approximation. Int. J. Non-Linear Mechanics, 30(5), pp. 741-757.

Poulin, F. \& Flierl, G., 2008. The stochastic Mathieu's equation. Proceedings of the Royal Society A: Mathematical, Physical and Engineering Sciences, Volume 464, pp. 1885-1904.

Primozic, T., 2011. Estimating expected first passage times using multilevel Monte Carlo algorithm. University of Oxford: s.n.
Sakawa, Y. \& Nakazumi, A., 1985. Modeling and Control of a Rotary Crane. Journal of Dynamic Systems, Measurement, and Control, p. 107:200.

Schuss, Z., 2010. Theory and Applications of Stochastic Processes - An analytical approach. Applied Mathematical sciences éd. New-York: Springer.

Sun, Z., Hou, N. \& Xiang, H., 2009. Safety and serviceability assessment for highrise tower crane to turbulent winds. Frontiers of Architecture and Civil Engineering in China, Volume 3, pp. 18-24.

Vanvinckenroye, H., 2015. Monte Carlo simulations of autorotative dynamics of a simple tower crane model. s.1., Proceedings of the 14th International Conference on Wind Engineering, Porto Alegre.

Voisin, D., 2003. Etudes des effets du vent sur les grues à tour. $\mathrm{PhD}$ thesis éd. Ecole Polytechnique de l'Université de Nantes: s.n.

Voisin, D. et al., 2004. Wind tunnel test method to study out-of-service tower crane behaviour in storm winds. Journal of Wind Engineering and Industrial Aerodynamics, 92(7-8), pp. 687-697.

Xu, X. \& Wiercigroch, M., 2006. Approximate analytical solutions for oscillatory and rotational motion of a parametric pendulum. Nonlinear Dynamics, 47(1-3), pp. 311-320.

Xu, X., Wiercigroch, M. \& Cartmell, M., 2005. Rotating orbits of a parametrically-excited pendulum. Chaos, Solitons \& Fractals, 23(5), pp. 1537-1548.

Yurchenko, D., Naess, A. \& Alevras, P., 2013. Pendulum's rotational motion governed by a stochastic Mathieu equation. Probabilistic Engineering Mechanics, Volume 31, pp. 12-18. 